\title{
Erhard Blankenburg Der Aufstand der kleinen Richter gegen die Korruption der großen Politik
}

Während Untersuchungsrichter in Italien, Frankreich und Spanien sich in den neunziger Jahren mit Korruptionsvorwürfen anf die Vorderbühne der Politik zu spielen wußten, werden entsprechende Ermittlungsverfahren in Deutschland überwiegend von den Staatsanwaltschaften eingestellt. Der ausschlaggebende Faktor für die politische Rolle, die die Justiz in einigen Ländern spielt, sind die Vollmachten von Untersuchungsrichtern oder auch Staatsanvoälten im Vorverfahren. Offensichtlich ist dabei das Zusammenspiel mit den Medien unverzichtbar, die dank der zugenommenen Konkurrenz aggressiver in investigativen Journalismus investieren. Kein Wunder, daß Politiker die Skandalisierung wiederum benutzen: In den Vereinigten Staaten von Amerika und in $R u ß l a n d$ verselbständigen sich Skandalvorvürfe zum routinisierten Mittel des personellen Machtwechsels.

Die Red.

\section{Die Ausgangsfrage}

Erzählende, moralisierende und skandalisierende Literatur über Korruption gibt es zu allen Zeiten. Gelingt es, ihre Wirkung bis zum effektiven Skandal zu steigern, dann kündigt sie soziale und politische Entwicklungen an - häufig lange bevor der ernsthafte Diskurs sie analytisch einholt. Es lohnt sich daher, sie zu lesen als moralisierenden Spicgel der Veränderungen politischer Regimes.

So kann die Skandalwelle um Bestechungsgelder bei der Beschaffung von LockheedFlugzeugen zu Beginn der 7oer Jahre als ein früher Bote der Globalisierung nicht nur des Welthandels, sondern auch der mit ihm verbundenen Korruptionsvorwürfe gesehen werden. Dem Lockheed-Skandal kommt das historische Verdienst zu, Konkurrenz- und Handelsregeln international reklamiert und damit die etablierten Klientel- und Finanzierungssysteme in zahlreichen Regimes illegitimiert zu haben: Prinz Bernhard der Niederlande, Presidente Leone in Italien, der japanische Premier Tanaka. In Japan und Italien sowie im niederländischen Königshaus war man seinerzeit höchst erstaunt, daß die ausländische Presse ein Verhalten anprangerte, das unter einheimischen Traditionen als völlig normal gegolten hatte. Es war das amerikanische Magazin Newsweek, das für die heimische Politikarena ein Bestechungskartell anprangerte, was durchaus unvorhersehbar in verschiedenen Geberländern Skandale entfesselte. Deren politische Systeme waren bis dahin skandalfrei gewesen, einfach deshalb, weil ihre klientelistischen Strukturen nie in Frage gestellt worden waren.

Der Lockheed-Skandal war ein gelungener Versuch, den Vorwurf der Korruption global zu benutzen und damit lokale Klientelsysteme verschicdenster Art zu Fall zu bringen, und er zeigte, daß dies mit Erfolg möglich war. Man kann es als Paradox ansehen, daß der universalistische Moralanspruch, der dem zugrunde liegt, von einem historisch bestimmten Interesse ausging: dem Interesse eines Welthandels an der Durchsetzung globaler Konkurrenzregeln unter Benutzung der sich globalisierenden 
Kommunikationsmedien. Wieder einmal erwies sich damit, was Carl J. Friedrich' beschäftigt hat: daß zwar der Vorwurf der Korruption zu allen Zeiten erhoben wurde, das Phänomen jedoch historisch keineswegs stets das gleiche gewesen ist. Verfehlt sind daher auch die Versuche, das Vorkommen von Korruption ätiologisch zu erklären, wie dies etwa van Klaveren ${ }^{2}$ tut mit seiner These, daß das Fehlen einer moralisch kontrollierenden Mittelschicht für das Aufkommen korrupter Praktiken verantwortlich sei. So sehr dies bei einigen, auch von den Zeitgenossen als korrupt angesehenen Erscheinungen plausibel sein mag: etwa bei der Bereicherung von kolonialen Beamten der Ostindischen Kompanie, so wenig deckt es den ehrgeizigen Versuch, das Phänomen historisch auf einen gemeinsamen Nenner zu bringen: so etwa wenn man erklären will, wieso der prominente High Chancellor Francis Bacon wegen seiner korrupten Gerichtspraxis zu Fall gebracht werden konnte. Von der Historie gezwungen muß auch van Klaveren relativieren, etwa wenn er den fiskalischen Ämterkauf unter Louis XIV. als legale Einkunftsquelle seiner absoluten Herrschaft und Mittel der Kontrolle von ungeregelter Korruption schildert. Erst die Französische Revolution schaffte den legalen Ämterkauf (mitsamt dem Adel) ab. Seine These trifft dann wieder zu, als die aufkommende Bürgerschicht das Zerrbild des korrupten Ancien Regime benutzte, um ihre Vorstellungen von einer rechtsstaatlichen Verwaltung mit dem Leitbild des legal gebundenen Beamten zu legitimieren. Aus der Einsicht, daß die Definition dessen, was als korrupt angesehen wird, sich mit den Zeiten (daß heißt auf die politische Korruption bezogen: mit den Regimes und deren Oppositionen) völlig verändert', folgt, daß vor jeder Untersuchung des Vorkommens von Korruption die Analyse derer stehen muß, die sie als solche definieren. Verführerisch ist dabei die Versuchung, nur die Fälle in die Betrachtung einbeziehen, die erfolgreich zu einem Skandal geführt haben. Wie bei allem moralisch verwerflichen Handlen ist die Dunkelziffer der Fälle weit größer, bei denen Empörungen der Opfer erfolglos blieben und kein Skandal zu entfesseln war. Eine konsequent konstruktivistische Theorie der Korruption muß also die schwierige Frage beantworten, warum zu welcher Zeit welcher Skandal erfolgreich sein und welche Definition von Korruption sich etablieren konnte.

Dies gilt auch für die Betrachtung gegenwärtigcr Korruptionsvorwürfe, deren Moralisierung und deren Instrumentalisierung. Auch wenn wir dabei von einem Auslöser wie dem Lockbeed-Skandal und seiner Konstellation politischer Interessen und Kräfteverhältnisse ausgehen, zeigen sich doch (nach C. J. Friedrich: pathologische) Lernprozesse, mit denen sich die Skandale um Korruption seit den siebziger Jahren verändert haben.

1 Carl ]. Fricdrich (1972) The Pathology of Politics: Violence, Betrayal, Corruption, Secrecy and Propaganda, New York: Harper \& Row.

2 Jacob van Kiaveren (1957-1960) Dic historische Erscheinung der Korruption, Aufsatzreihe im Archiv für Sozial- und Wirtschaftsgeschichte 44-47.

3 Eine Ubersicht der wissenschaftlichen Literatur zu jener Zeit findet sich bei Arnold Heidenheimer (1970) Political Corruption, derselbe erweiterte diese zu einer umfassenden Dokumentation zusammen mit Michael Johnston/Victor LeVine (1998) Political Corruption, New Brunstvick: TransactionPubl. 
Die neunziger Jahre erleben nicht nur in Italien, auch in Frankreich oder Rußland, Berlin oder Frankfurt eine Welle der Skandale und Verfahren wegen Korruptionsvorwürfen. Wurden sie bislang ohnmächrig und achselzuckend mit einem zynischen Ausdruck der Politikmüdigkeit hingenommen, überrascht jetzt, daß die Vorwürfe zu Verfahren und gelegentlich sogar zum Erfolg führen, ja daß sie gar zum Zusammenbruch eines Regimes beitragen können. Allerdings mahnt der Lärm der Skandale auch zur Vorsicht: Bei den Staatskrisen in Indonesien und der Sowjetunion, in Japan oder Italien spielten Korruptionsvorwürfe mit, um die Legitimität des untergehenden Regimes zu erschüttern, aber in keinem dieser Fälle gaben sie den Ausschlag für den Zusammenbruch. Es muß schon eine andere (meist wirtschaftliche) Krise hinzukommen, ehe ein korruptes System wirklich zusammenbricht. In der Turbulenz krisenhafter Ereignisse allerdings werden die unterliegenden Schwächen eines Regimes oft übertönt von dem jeweils auslösenden Skandal.

Die meisten Skandale bcginnen harmlos. Als Beispiel mag der Fall Craxi in Mailand dienen, der 1992 innerhalb eines Jahres das Partciensystem Italiens zum Einsturz brachte. Er beginnt mit der Verhaftung Mario Chiesa's wegen einer kleinen Unterschlagung. Der Verwalter sozialistischer Wohnungen und Waisenhäuser, und dabei auch Summenbesorger der sozialistischen Partei und Annehmer der Parteifinanzierung, wird von seiner Partei fallen gelassen, der mächtige Parteiboss Craxi nennt ihn verächtlich meinen kletnen mariolo". Chiesa sitzt in Untersuchungshaft und wehrt sich empört. Er zieht andere mit herein. Die Ermittlungen weiten sich aus und werden zum Vorwurf der Korruption. Schon ist ein kleines Netzwerk vor Gericht gestellt. Als die Bosse jetzt versuchen, ihn herauszuhauen, kommen sie selbst in die Angriffslinie. Sie versuchen es mit einem Gegenangriff auf die Presse, die Justiz und den Gegner selbst. Die Sache eskaliert, und jetzt haben wir den schönsten Skandal, damit auch eine Ervweiterung der ursprünglichen Vorwürfe. Aus dem Verdacht einer kleinen Unterschlagung ist die Anklage eines "ganz und gar korrupten Regimes" geworden.

Beachtlich ist, wie sich die Definitionen mitlaufend verändern: Erst im Laufe des Skandals wird definiert, was als "Korruption " gebrandmarkt werden soll. Der Erfolg des Skandals definiert die Korruption.

Zentral ist dabei, wer an dem Definitionsvorwurf mitwirkt. In Italien ebenso wie in Frankreich oder Spanien der neunziger Jahre kommen Skandal und die Definition von Korruption als Wechselwirkungen zwischen politischen und justiziellen Institutionen und diesen wiederum mit Medien und Geschäftswelt zustande. Sie lassen sich anschaulich in einem Schema verschiedener sozialer Arenen und ihrer Interaktionen darstellen:

\begin{tabular}{|cc|}
\hline & $\begin{array}{c}\text { Interakion der Macht } \\
\text { Politik }\end{array}$ \\
Diskurs des Rechts & \multicolumn{1}{c|}{ Diskurs öffentlicher Moral } \\
Justiz & Medien \\
& $\begin{array}{c}\text { Interaktion des Geldes } \\
\text { Ökonomie }\end{array}$ \\
\hline
\end{tabular}

Das Schema der Arenen dient der Darstellung von Kopplungen, mit denen Politik und Recht, Geld und Moral zusammenwirken. Jede der Arenen hat ihre eigene Logik, aber erst das Zusammenwirken erklärt die Wahrscheinlichkeit von Skandalen und damit auch den reklamierten Umfang der politischen Korruption. 

verändert:

- An die Stelle der ideologisch gefestigten Parteien mit klassenmäßig definiertem Anhängerstamm sind von Wechselwählern abhängige Wablkampfmaschinen getreten. Politiker müssen für ihre Wahlkämpfe immer höhere finanzielle Verpflichtungen eingehen. Je nachdem wie viele Sponsoren sie dazu bemühen, müssen sic nach der Wahl zurückzahlen: stets durch Einlösen von politischen Versprechungen, aber auch durch Ämter, Staatsaufträge und Zugang zu Entscheidungen, die ihre überlebensnotwendige Klientel bei Laune halten. In den USA gilt ein solches spoils system< traditionell zu den etablierten Regeln der Demokratie, was durch das Übermaß an Regelungen, mit denen Washington die sppoilss transparent zu machen sucht, nur noch betont wird. Es ist ein strukturelles Problem der amerikanischen Wahlkampf-Parteien, das nun auch viele europäische Länder ereilt, in denen bislang ideologische Parteien mit ihrem festen, klassenbedingten Anhängerstamm die Struktur bestimmten. ${ }^{4}$ Das Geschäft der Politik ist korruptogener geworden, damit allerdings zugleich auch die stets drohende Gefahr, Opfer von Skandalisierungen zu werden.

- Das Skandalisieren ist das Geschäft der Medien. Ihr zunehmender Konkurrenzdruck zwingt sie zur Jagd nach immer neuen Sensationen, die sie möglichst mit Informationsvorsprung einige Zeit ausbeuten können. Seit dem Lockheed-Skandal und dem Watergate-Skandal zu Beginn der siebziger Jahre haben politische Magazine in Teams für aufwendige Langzeitrecherchen (investigatory journalism) investiert. Fernsehanstalten und auch unabhängige Journalistenbüros sind in den letzten fünfzehn Jahren gefolgt und führen heute einen gnadenlosen PapparazziKampf um erfolgreiche Skandale auf. Mit ihren Enthüllungen kann man sie als Moralunternehmer bezeichnen, die oft erfindungsreich neue Vorwürfe ersinnen und damit schlicht ausprobieren, welche Themen sich zur öffentlichen Empörung eignen könnten.

- Neu in diesem Spiel zwischen den Arenen ist die Prominenz der Justiz. Skandale bedienen sich mit Vorliebe rechtlicher Verfahren, vornehmlich natürlich vor den Strafgerichten, weil diese moralerhöhende Symbolik und Legitimität verschaffen. Aber traditionell tun sie dies erst in späteren Phasen, wenn ein Skandal schon Fahrt bekommen hat. Daß juristische Verfahren oft lange dauern, kann den Medien recht sein; daß sie mit Beweisschwierigkeiten kämpfen, erhöht die Dramatik, und es stört wenig, wenn sie zuweilen mit Freisprüchen enden. Das Verfahren an sich ist ja schon die Strafe.

In den lateinischen Ländern: Italien, Frankreich und Spanien haben Staatsanwälte und Untersuchungsrichter darüber hinaus eine bislang ungekannte Rollc als Skandalauslöser entwickelt. Ermutigt durch einzelne aufsehenerregende Prozesse setzten sie seit den späten 8oer Jahren die außerordentlichen Mittel ein, die sie im Ermittlungsverfahren haben: In Frankreich wurde die Verhaftung eines Unternehmers wegen unzulässiger Entlassungen von der linken Richtergewerkschaft als Pioniertat gefeiert; in Mailand lösten Haussuchungen und später auch Verhaftungen von prominenten Politikern und Geschäftsleuten einen heftigen Streit zwischen Justiz und Parteien aus. Die spektakulären Aktionen blieben der Presse nicht verborgen und riefen Horden von Papparrazzi auf den Plan, und selbst wenn auf anschließend unumgänglichen Pressekonferenzen die Unschuldsvermutung hoch gehalten wurde, war der Skandal natürlich in Gang gesetzt. Ermitt-

4 Vgl. D. Della Porta/ A. Piazyorno (1996) The Busıness Poluncians: Relections from a Study of Political Corruption, in: Juurnal of Law and Socicty 23, 73-94. 
lungen wurden zum moralischen Prozeß, und die schlichte Skandal-Moral zur Strafe.

Theoretisch anspruchsvoll könnte man die Analyse der Arenen als Systemtheorie mit relativ geschlossenen Welten und strukturellen Kopplungen aufpurzen. Man kann sie sich wahlweise als Diskurs oder auch als Interaktionssystem vorstellen. Die unterschiedliche Benennung deutet nur an, mit welchen Mitteln sich im jeweiligen System Macht in erster Linie äußert. Die Akteure können versuchen, von der einen Arena in die andere überzutreten, etwa indem sie (mit hohem Anspruch auf Legitimität) Politik zu verrechtlichen oder Recht zu moralisieren suchen, oder (mit schlechten Legitimitätschancen) Medien zu beherrschen, politische Entscheidungen abzukaufen oder Richter zu bestechen versuchen.

Dabei folgt der Begriff der Korruption der jeweils sozial erwünschten Konstruktion. Im Gegensatz zu der Literatur der fünfziger Jahre können wir nicht mehr von der Korruption an sich sprechen, sondern müssen uns anschließen an das Konzept, das im Gefüge der Interaktionen und Diskurse als solche definiert wird. Die Frage, die sich dementsprechend stellt, ist nicht, wie korrupt ein Regime möglicherweise ist, sondern unter welchen Umständen der Definitionsversuch, daß ein Regime korrupt sei, erfolgreich ist und wie weit dies nicht gelingt.

Damit kommen wir zurück zu der Ausgangsfrage, unter welchen Bedingungen Skandale erfolgreich sein können, also zum Zusammenbruch eines korrupten Systems führen können. Es zeigt sich, daß dies nur möglich ist, wenn zu den Skandalen institutionelle Veränderungen im System der politischen, rechtlichen und moralischen Arenen hinzukommen.

Die hohe Schule des skandalösen Theaters findet man noch immer in Italien. Seit $199 \mathrm{I}$ hat sich das System der politischen Akteure und ihrer Partien völlig verändert, unter Wahrung jedoch der gewohnten klientelistischen Verhaltensmuster. Etrwas für italienische Zustände Unglaubliches ist geschehen: Fin kleiner ungerwöhnlich puritanischer Staatsan walt hat die gesamte Presse Italiens mobilisiert, sein Skandal fällt in eine Zeit von Neuwahlen, in denen die etablierten Parteien vernichtend geschlagen werden, der Staatspräsident Cossiga erklärt mit dem Ende des Kalten Krieges auch für Italien eine neue Ära als gekommen und tritt frühzeitig ab, ein neues Wahlgesetz beraubt die alte Garde der Parteien ihrer sicheren Positionen.

Das neue Kabinett unter Berlusconi unternimme noch einen Versuch, sich an die Spitze der Kampagne gegen "die schmutzigen Hände« zu stellen, indem es strenge Anti-Korruptionsregeln präsentiert, gepaart allerdings mit einem Dekret für die Amnestie vergangener Korruptionsvorwürfe. Der neue Präsident Scalfaro verweigert jedoch dic Lnterzeichnung des Dekrets, und im März 1993 folgen weiter Rücktritte von Ministern und Managern der großen Staatsbetriebe.

Der kleine Staatsanwalt Di Pietro wird zum Held der Nation und findet Nachahmer in der gesamten Justiz. Gegenangriffe bleiben nicht aus: Die Staatsanwaltschaft in Brescia ermittelt gegen Di Pietro, ihrerseits wegen Korruptionsvorwürfen, und demonstriert damit, wie sehr die italienische Justiz politisch polarisicrt ist. Jedoch: Das Verfahren muß eingestellt werden. Di Pietro geht zeitweise in die Politik.

Auch auf der Gegenseite treten der Korruption bezichtigte Geschäftsleute in die politische Arena ein. Berlusconi versucht sich 1994 als Erneuerer des Parteiensystems, scheitert allerdings nach kurzer, viel versprechender Regierungszeit, um nunmehr selbst von Skandalisierungen heimgesucht zu werden. Gegen zwei Verurteilungen wegen Korruption mit Gefängnisstrafen von mehreren Jahren ficht er zur Zeit in der Berufungsinstanz, ein drittes Verfabren läuft noch in der ersten Instanz. Im darauf entfesselten Gegenangriff stellt er sich als Opfer dar: Was konntc ein ehrlicher Kaufniann angesichts der Erpressung von Beamten, Steuerinspektoren und Parteien anderes tun, als die geforderten Bestechungen zu leisten? Nicht der Bestecher, sondern die Forderungen der zu Bestechenden tragen nach seiner Darstellung das korrupte System. Kräftig unterstützen seine Medien den Vorwurf, die Justiz verfolge einen »marxistischen « Staatstreich.'

5 Für eine gut informierte Analyse der italienischen Korruptionsaffairen siehe D. Della Porta/ A. Vanucci (1994), Corruzione politica e amministrazione pubblica. Ricurse, attori, mechnaimse. Bologan: ll Mulino. 
Angesichts der Szenen, die sich die neuen Parteien im nationalen Parlament liefern, kann man zweifeln, ob die versprochene Erneuerung der Parteien deren politische Struktur nachhaltig reformiert hat. Bleibend jedoch ist die Stärkung der Justiz, und dabei besonders der Stellung des Staatsanwaltschaft. Sie setzt die Stärkung der Justizautonomie fort, die (obwohl schon in der Verfassung von 1948 vorgesehen) 1959 eingeleitet wurde mit der Errichtung des Consiglio Superiore da la Magistratura. Dieser Oberste Rat hat zwar bis heute die Polarisierung der Richterschaft in politische Lager nicht verhindern können, schirmt aber die Rekrutierung und Beförderung von Richtern und Staatsanwälten wirksam gegen direkte Eingriffe der Exekutive ab. Dies gilt auch gemäß der Verfassung für die Staatsanwaltschaft, sodaß die Abschaffung des Instituts des Untersuchungsrichters mit der Strafprozeßreform von 1989 die Definitonsmacht auch politisch sensibler Strafsachen in die Hand einer unabhängigen Ermitclungsbehörde legt. ${ }^{6}$

Auch Frankueich hat in den neunziger Jahren den Einsturz von politischen Klientelsystemen erlebt, wenn auch zunächst nur in der Provinz. Die Namen von Justizhelden wie van Ruymbeke, Halphen und Jean-Pierre wurden über die Landesgrenzen hinaus bekannt. Seit r99 I tritt das satirische Magazin Le Canard Enchainé regelmäßig als Vorreiter auf, und seit 1994 lister die seriöse Le Monde 1994 jährlich über hundert Politiker auf, die in Untersuchungshaft sitzen. Auch hier sind die Vollmachten in der Ermittlungsphase entscheidend, die bei der französischen Staatsanwaltschaft allerdings traditionell noch den Eingriffsmöglichkeiten des Justizministers unterliegen. Daher wurden auf der nationalen Ebene politisch sensible Verfahren regelmäßig eingestellt; die Skandale in der Provinz dagegen weiteten sich aus. Seit 1997 allerdings haben sich die Bedingungen des politisch motivierten Stillhaltens verschlechtert: Unter der Polarisierung der Kohabitation drohte die Skandalisierung sogar gegen den Präsidenten der Nation gerichtet zu werden. Der Verfassungsrat schob dem im Januar 1999 einen Riegel vor, indem er die Immunität des Präsidenten bekräftigte. Richter jedoch sind auch in Frankreich unabhängig, und da gemäß der lateinischen Tradition des Strafprozeßrechts das gerichtsrelevante Stadium der Ermitclungen beim Untersuchungsrichter (und verstärkt noch beim sconseiller in der Berufungsinstanz) liegt, haben diese ihre Skandalfähigkeit entwickelt. Sie können Verhaftungen vornehmen, sogar eine Beugehaft veranlassen, was vor der Neugier der Medien kaum verborgen bleibr. Auch wenn sie (wie die beiden crsten der obengenannten) gegenüber den Medien äußerst zurückhaltend bleiben, ist eine Instrumentalisierung der Medien nicht ganz von der Hand zu weisen. Bei einigen Skandalen (wie der Affaire um finanzielle Manipulationen des URBA Konzerns 1991) nutzen Untersuchungsrichter ihr Recht auf Haussuchung und persönliche Augenscheinnahme explizit, um die Ermittlungen gegen eine Einstellung durch die Vorgesetzten der Staatsanwaltschaft zu schützen. Unter der Decke der justiziellen Verschwiegenheit liefern sich unabhängige Richter mit den hierarchisch abhängigen Statsanwälten manches juriscisch ausgefeilte Verfahrensspiel. ${ }^{7} \mathrm{Da}$ kann eine Unterstützung durch Medien, wenn sie denn ohne offenen Geheimnisbruch zustande kommt, der Effektuierung der Strafverfolgung helfen.

Allerdings eskaliert ein Verfahren durch ein solches Spiel mit der Arena der Medien zu einem öffentlichen Skandal und erhält damit den Charakter der Vorverurteilung. Gegenangriffe auf die Justiz, vor allem von betroffenen Verdächrigten, sprechen denn auch von einem "Staatsstreich der kleinen Richter? ${ }^{8}$ Sie erhalten Nahrung durch die

6 Siehe für alle hier genannten Länder die vergleichende Darstellung bei Carlo Guarnieri/Patrizia Pederzoli (1996) La Puissance de Juger, ch $\mathrm{r}$ et 2, Paris: Michalon.

7 Ausführlicher die Analyse auf Basis von Richter-Interviews von Violaine Roussel, Les magistrats dans les scandals politiques, in: Revue française de la science politique 48,2 (1998) $245-273$.

8 Vgl. Denis Robert, La justice ou le chaos, Paris: Stock 1996. 
politische Aktivierung von Richtern der 1968 er Generation in den Richtergewerkschaften und dem r 990 gegründeten Forum de la Justice.

In Spanien spielt Richter Garzon eine ähnliche Rolle wie die Justizhelden in Italien und Frankreich. Als nationaler Untersuchungsrichter (juéz central de instrucción bei der Audiencia Nacional) reicht seine Kompetenz von der Drogenfahndung über organisiertes Verbrechen und Korruption bis zum Kampf gegen den Terrorismus. Die von ihm untersuchten Skandale trugen maßgebend zum Fall der sozialistischen Regierung bei, unter der sich - zunächst in der Guardia Civil und im Innenministerium unter dcm Schutz der Terrorismusbekämpfung mit ihren verdeckten Aktionen und Geldern - eine Struktur der Korruption entwickelt hatte?. Auch hier entfalteten im Zusammenspiel von Ermittlungsrichter und Medien die Vorverurteilungen ihre Wirkung.

Die Konstellation einer sich politisch emanzipierenden Justiz gegenüber einer traditionell klientelistischen Politk beruht in allen drei Ländern auf den sozialen Veränderungen unter der neuen Generation von Richter(in)en und Staatsanwält(in)nen, die durch das Größenwachstum der Justiz noch akzentuiert werden. Die drei lateinischen Länder in Europa kannten eine historisch starke Bindung der Justiz an das politische Establishment: Vor zwanzig Jahren war die spanische Justiz noch faschistisch dominiert, die italienische polarisiert zwischen Christdemokraten und linken Parteien, und die französische fcst orientiert an den regionalen und nationalen Eliten. Das Aufbrechen dieser Regimes und die Ablösung ihrer Eliten bot der Justiz Gelegenheiten, ihre Unabhängigkeit als dritte Macht institutionell zu festigen; ein Ausdruck hiervon ist die Errichtung von ,Obersten Nationalen Richterräten. In der Skandalpolitik allerdings sind es stets einzelne Richter oder auch Staatsanwälte, die sich publikumswirksam gegen politische Korruption und Steuerhinterziehung zur Wehr setzen. Der Genfer Generalstaatsanwalt Bernard Bertossa hat 1995 die Symbolfiguren aus diesen Ländern zu eincr gemeinsamen Erklärung zusammengebracht. Mit ihrem Appell von Genf versuchten sie 1995, eine gemeinsame Front der Justiz als moralischer Instanz gegenüber den illegalen Finanzen von korrupter Politik und steuerhinterziehender Wirtschaft aufzurichten. ${ }^{\circ}$

Natürlich blieb auch der Gegenvorwurf, daß es diesen kleinen Richtern an demokratischer Legitimität fehle, nicht aus ". Je wirksamer die politische Rolle der Justiz wird, desto eher wird sie auch an deren Legitimitätsvoraussetzungen gemessen. Aktive Skandalisierung gefährdet ihre privilegierte Unabhängigkeit; Zurückhaltung gegenüber den Medien ist also angesagt. Die damit verbundene politische Disziplinierung gelingt eher dort, wo keine unabhängigen Staatsanwälte oder Untersuchungsrichter, sondern eine hierarchisch geleitete Behörde die Ermittlungen in eigener Hand behält.

Beispielhaft ist dies in Deutschland der Fall. Die interne Kontrolldichte ist groß, erkenabar daran, daß die Bürokratien und ihre Rcchnungshöfc viele Ermittlungen führen. Aber sie halten gegenüber der Öffentlichkeit weitgehend dicht; selbst beim Groß-Ausverkauf der DDR-Ökonomie durch die Treuhand-Gesellschaft sind Details der Scheingeschäfte und Bevorteilungen kaum ans Tageslicht getreten. ${ }^{12}$ Auch die Staatsanwaltschaft führt viele Ermittlungen und hat sich zeitweise mit Informationen

\footnotetext{
9 So wic zu Zeiten Gonzalcz: II Mundo als Blatt der Opposition die Skandale in die Öffentlichkeit spielte, so ist es nun El Pats als Oppositionsblatt gegen Aznar.

ro Siche die Dokumentation bei Denis Robert (Fn. 8).

I Für cine Polemik gegen die zunehmende Macht der Richter als moralische Wachhunde vgl. Eric Zemmour (1997) Le Coup d'Etat des Juges, Paris: Grasset.

12 Immerhin hat eine parlamentarische Enquête 1998 als Schätzung des Umfangs einen Betrag von Bo Mitlionen angegeben.
} 
über deren steigenden Umfang an die Presse gewandt. In den goer Jahren inszenierten einige Staatsanwälte (allen voran der Frankfurter Staatsanwalt Schaupensteiner) eine gezielte Thematisierung ${ }^{13}$ der verbreiteten Korruption in städtischen Ämtern bei öffentlicher Auftragsvergabe. Auffallend jedoch ist, daß es selten zu richterlichen Verfahren kam, da die meisten Verfahren an den Schwierigkeiten ausreichender Beweisführung strandeten. Die Skandalisierung der Ermittlungsbehörden galt ihrem eigenen Versagen, mit dem sie den Gesetzgeber zur Erweiterung ihrer Ermittlungsbefugtheiten bewegen wollten. ${ }^{14}$. Der Erfolg stellte sich 1997 mit dem Anti-Korruptionsgesetz ein. Danach war wieder Ruhe. Die Skandalwelle hatte ihr vorläufiges Ziel erreicht.

In den 8oer Jahren war dies zeitweise anders: Beim Aufdecken der Flick-Affaire war die Verschlossenheit der staatsanwaltlichen Information durchbrochen. Der Vorwurf, daß die nordrhein-westfälische Justiz in Bonn in Opposition gegen den Filz von Christdemokraten und Liberalen die Enthüllungen des Magazins >Der Spiegek unterstützte, blieb unbewiesen, aber daß Informationen aus Ermittlungsakten an die Öffentlichkeit gelangten, war Erfolgsbedingung der Skandalisierung. Der Ablauf der Korruptions- und Betrugs-Affairen der 8oer Jahre, die zum Einsturz der Parteifinanzierung der Regierungsparteien, aber auch zum Zusammenbruch des Wirtschafts-Imperiums der Gewerkschaften mit dem Baukonzern Neue Heimat und der gewcrkschaftlichen Genossenschaftsbank führten, zeigt, daß unter den deutschen Medien durchaus Meister der Skandalregie zu finden sind"s - auch ohne daß sie sich der Öffentlichkeitsarbeit von Justizhelden versichern können.

\section{Die Eigendynamik der Skandalindustrie}

Obwohl also das Zusammenspiel von Medien und Justiz nationalen Bedingungen unterliegt, nimmt es doch teil an der Globalisierung von Normen. Die oft platte Moral der Korruptions-Skandale läßt sich leicht instrumentalisieren, um politische Gegner zu beschädigen oder gänzlich von der Arena zu entfernen. Groß ist daher die Versuchung, das Instrument der Skandaliserung zu inflationieren.

Nachdem der Watergate-Skandal die Macht der Medien in der amerikanischen Poiltik demonstriert hatte, entwickelten sich Nachahmer-Effekte. In der ganzen Welt bauten Fernsehanstalten, Tages- und Wochenzeitungen ibre Stäbe für aufwendige Recherchen aus. Investigatory journalism ist in den vergangenen zwanzig Jahren $z$ u einer Skandalindustrie geworden.

Das sichtbarste Beispiel für die Skandalinflation bieten die Vereinigte Staaten von Amerika. Die Finanzierung der Wahlkämpfe - von der nationalen bis zu der lokalen Ebene, Plebiszite und Nebenwahlkämpfe von Richtern und Staatsanwälten eingeschlossen - bringt ein spoils system mit sich, das jeden Wahlkämpfer an Interessengruppen bindet und zum Anwerben von Sponsoren zwingt, die natürlich später

13 Christian Höffling an der Universität Bremen hat die Karrierre des Themas von den ersten Veröffentlichungen von SrA Schaupenstein 1990 in der Fachzeitschrift Krmmalistik bis zur Ubernahme in den Massenmedien Spregel, Stern, Focus und Die Woche zwischen Dezember 1994 bis Juli r 995 aufgezeichnet. Manuskript Bremen 1997.

I4 Trotz steigender Zahlen von Ermittlungsverfahren blieben Anklagen und Verureilungen wegen Bestechung, Amtsmißbrauch erc. selten. Siche Werner Rügener (1997) Die deutsche Justiz und dic Korruption, in: Kritische Justiz 30, 458-474.

is Ausfuhrlicher in meinem Beitrag (1990) Korrupcion und Skandal - Zwei Seiten derselben Medaille in: Hans Oswald (Hrg.) Mache und Recht, Festschrift fúr Heinrich Popitz zum 6s. Geburtstag, Opladen: Westdeutscher Verlag, I4 I-I I 4 . 
entlohnt werden müssen. Die Klientelsyteme der machine politics, mit der vor allem örtliche Parteien sich an der Macht halten, erscheinen - zumindest nach europäischen Maßstäben - als eine strukturelle Vorraussetzung für Korruption. ${ }^{16}$ Umfragen allerdings zeigen eine für Europäer erstaunliche Toleranz mit dem System der Ämterpatronage und öffentlicher Auftragsvergabe: Amerikanische Abgeordnete bezeichnen sie als Elemente einer demokratischen Normalität, wenn sie auch - zugegebenkorruptogen seien ${ }^{17}$; und Wähler entziehen ihren Kandidaten keineswegs ihre Stimmen, nur weil diese in einen Skandal verwickelt wären. ${ }^{18}$

Skandale sind in der amerikanischen Politik Produkte der Medien und unterliegen deren Gesetzmäßigkeiten. Wie andere Konflikte auch - sei dies die Wahlkampffinanzierung, die Homosexualität von Bischöfen oder der Sex des Präsidenten werden sie schnell auf die juristische Bühne gespielt. Die Justiz nimmt als Legitimitätsbeschaffer vielfältiger Moralkampagnen an der Inflation von Skandalen teil. Skandale - auch ohne Korruption - sind während der Clinton Präsidenschaft zu einem populistischen Spiel instrumentalisiert worden - mit dem Effekt einer Demoralisierung der Politik.

Als Nachahmer-Effekt - oder auch als Globalisierung westlicher Legitimitätsnormen - müssen wir auch die Korruptionsaffairen im nach-sowjetischen Rußland sehen:

Dort, in Rußland, wirkt das System der allgegenwärtigen Korruption als jederzeit einsetzbare Normfalle, die von allen Seiten als Mittel zur Ablösung von Personen und ganzer Klientelgruppen eingesetzt werden kann. Schon in der Sowjetunion hatten Chrustschow und Andropov ihren Amtsantritt mit großen Anti-Korruptionskampagnen begonnen und damit den Regierungsapparat von Gegnern gesäubert und eigene Gefolgsleute an ihre Stelle gesetzt. In einer Planwirtschaft, die nur durch Tauschsysteme des Schwarzhandels aufrecht erhalten werden konnte, war ein Korruptionsvorwurf jederzeit und gegen jeden möglich. Die Doppelmoral der legalen und illegalen Märkte wurde zum Mittel des regulären Personalwechsels.

Jeltzin benutzte dieselbe Technik 1992/93 in seinem Kampf gegen Rutschkoi, den er im Oktober 1992 zum Vorsitzenden einer "Interdepartmentalen Kommission zur Bekämpfung von Kriminalität und Korruption* ernannt hatte. Im April 1993 übernahm er selber den Vorsitz dieser Kommission, und im August kündigte er unerwartet in einer Pressekonferenz an, daß er dem Moskauer Staatsanwalt Auftrag gegeben habe, gegen Rutschkoi und seine Bande wegen Verschieben von Geld auf Schweizer Konten zu ermitteln. Ähnliche Vorwürfe gegen präsidentielle Mitarbeiter von seiten des obersten Sowjet waren dem vorausgegangen. Neben dem militärischen Coup, der schließlich zur Schlacht um das "Weiße Haus in Moskau führte, spielte sich der Machtkampf zwischen Jeltzin und der Staatsduma als Konkurrenz um die Besetzung des Generalstaatsanwalts ab. Die Gruppe um Präsident Jeltzin gewann diesen Kampf, und damit die Definitionsmacht über den Korruptionsvorwurf. ${ }^{19}$

16 Für die frühe Thematisierung lokaler amerikanischer Korruption vgl. den Reader von John Gardiner/ David Olson (1974) Theft of the City, Bloomington: Indiana U. Press. Klassisches Vorbild ist das ward system der Demokratischen Partci in Chicago zu Zeiten des Bürgermeister Richard Daley in den 6oer/70er Jahren. Unter der umfangrcichen Literatur bieter einen guten Einblick Milton Rakove (1975) Don'c Make No Waves, Don't Back No Looscrs, Bloomington: Indiana U. Press.

17 Vgl. die Umfragen in Nachwirkung der Absetzung Nixon's bei Edmund Beard/ Stephen Horn, Congressional Fthics: The View from the House. Washington: Brookings 1975 ; sowic Joho Peters/Susan Welch, Politics, Corruptionand Political/Culture: A View from the State Legislature, in: Am. Politics Q. II,4 507-517, 1983 .

18 Barry Rundquist et al. (1977) Corrupr Politicians and their Electoral Support, Am. Pol Science Rev. 954963

19 Quelle: Hans Oyersloot (1996) Rusland deze jaren: hervorming door corruptic, Rijks Universiteit Leiden. 
Der Anteil der Korruptionsvorwürfe an der Skandalindustrie ist je nach politischer Kultur verschieden. Skandale lassen sich um beliebige landeseigene Hysterien entfesseln: sei es wegen Sex in Amerika, den Royals in England oder westlichen Sitten in islamischen Ländern. Aus ihrer Machbarkeit folgt, daß wir vom Ausmaß der Skandale nicht auf einen irgendwie meßbaren tatsächlichen Sittenverfall schließen können. Das gilt auch für Korruptionsskandale: Ihre Auslösung verhält sich unabhängig von jedem irgendwie vergleichbar meßbaren Ausmaß und Gelegenheit zur Korruption. Der Schluß von den bekannten Skandalen auf das unbekannte Ausmaß könnte fehlgeleitet sein.

Dennoch wäre es falsch, die Relativierung so weit zu treiben und zu sagen, Korruption gebe es doch überall, gleich wie das Regime auch sei. Trotz aller Schwierigkeiten objektiver Messung sind Unterschiede nicht nur in der Form, die Korruption jeweils annimmt, sondern auch in deren Ausmaß nicht abzustreiten. Entsprechend den globalisierten Interessen sind es in jüngster Zeit Organisationen wie die Weltbank und der International Monetary Fund (IMF), die Korruption als Feind des internationalen Wettbewerbs ausgemacht haben.

Das bringt uns zu der vierten Arena des obigen Schemas: der Geschäftswelt, die zunehmend ibre Regeln international fairer Konkurrenz und freien Marktzugangs mit Sanktionen zu bewehren sucht. Sie hat hierfür die internationalen Handelsverträge auf ihrer Seite:

- wie die der Europäischen Gemeinschaft, die mit ihren Vorschriften der europaweiten Ausschreibung unter gleichen Bedingungen mit so mancher Gewohnheit nationaler und auch regionaler Protektion aufräumt,

- oder die internationalen GATT Vereinbarungen, die der amerikanischen Regierung erlauben, auf die internationale Vergabe von Bauaufträgen zu dringen, und damit zum Beispiel um japanische Bauaufträge zu konkurrieren, die bis dato ein Schutzpark des pork barrel von hohen Beamte der MITI waren ${ }^{20}$,

- oder auch den Druck von Weltbank und IMF, die ihre Kreditvergabe in Indonesien und anderswo an Bedingungen knüpfen, mit denen sie hoffen, den Nepotismus zu beenden.

Die Definition von Korruption, von der dabei ausgegangen wird, ist zunächst schlicht als Gegenmodell des freien Marktes entwickelt. Die Häufigkeit korrupter Erpressung, wie etwa die Aufforderung, Bestechungsgelder zu zahlen, um an Aufträge zu kommen, lassen sich objektiv messen, wenn auch mithilfe von subjektiven Einschätzungen. Dies tut der pfiffige Versuch eines ehemaligen World Bank Funktionärs ${ }^{21}$, der das Befragungsinstrument einsetzt, um unter den meist unfreiwilligen Bestechern Informationen über die zu Bestechenden einzuholen. Die "bitlist of shame«, die Transparency International auf dieser Basis aufstellt, entfaltet ihre Wirkung über weltweite Medien. Zusammen mit der fühlbarcn Sanktion des Geldes entfalter diese Strategie der Beschämung durchaus ähnliche Abschreckungseffekte wie die Skandalisierung einzelner Ereignisse.

Wissenschaftlich allerdings sind die Einschätzungen der betroffenen Geschäftsleute noch unbefriedigend, da sie der Erklärungen im Kontext der jeweiligen politischen, moralischen und Rechtskultur bedürfen. Der Transparency Index mißt vor allem die externe Reputation von Ländern in bezug auf Bestechungsgelder, die bei staatlicher

20 Vgl. Brian Woodell (1996) Japan under Construction: Corruption, Politics and Public Works, Berkeley: U. of California Press.

2I Vgl. Peter Eigen, Combatting Corruption in the Free World, Transparency International Berlin. 
Auftragsvergabe verlangt werden. Angesichts der enormen Summen, die in vielen Empfängerländern von Krediten der Weltbank mim Laufe der Transaktion versikkern «, ist dies ein plausibler Ausgangspunkt. Allerdings führt jede Analyse der Formen und Gründe dieses Versickerns auf die klientelistischen Überlebensbedingungen in Ländern, in denen Herkunft, extensive Familienverbände und Seilschaften Wirtschaft und Gesellschaft zusammenhalten. Viele Kenner von Entwicklungsebenso wie von post-kommunistischen Ländern ${ }^{22}$ sehen daher jeden individuellen Korruptionsvorwurf als wirkunglos an und sprechen allenfalls zwischen Anführungszeichen von "struktureller Korruption" ganzer Regimes. World Bank, IMF und andere Kreditgeber richten sich infolgedessen auch nicht nur auf die Skandalisierung von korrupten Praktiken, sondern versuchen, den institutionellen Kontext, der Korruption fördert, in ihre Strategie einzubeziehen. Damit sind alle Gelegenheitsstrukturen politischer Korruption im Bild: die Parteifinanzierung, die klientelistische Ämter- und Auftragspatronage, die Kontrolle von Rechnungshöfen, die Freiheit der Presse und das Funktionieren demokratischer Korrekturen insgesamt.

Hierbei steht der Begriff Korruption wie schon bei Montesquieu und Rousseau ${ }^{23}$ für den allgemeinen Sittenverfall der Gesellschaft. Er dient der Errichtung eines Gegenmodells der guten Gesellschaft. Das Utopiewort heißt dann "good governance "24 und schließt den moralischen Kontext freier Marktwirtschaft ebenso ein wie die Indikatoren einer politischen Zivilgesellschaft.

\section{Marc Hudy \\ Elektronisch überwachter Hausarrest}

Befunde zur Zielgruppenplanung und Probleme einer Implementation in das deutsche Sanktionensystem

Nach Modellversuchen in Großbritannien, Schweden und den Niederlanden wird die Einführung des aus den USA hervorgegangenen elektronisch überwachten Hausarrestes auch hierzulande kontrovers diskutien. Hohe Erwartungen hinsichtlich einer kosteneffizienten, sicheren und dennoch hunanen Haftvermeidung werden mit dieser neuen Sanktionsform ebenso verbunden wie Befürchtungen bezüglich einer technologisch unterstützien Ausweitung staatlicher Sozialkontrolle. Bereits vorhandene Befunde, Erfahrungen und Probleme bleiben dabei in der öffentlichen Diskussion weitgehend unbeachtet.

Am Beginn dieser Untersuchung steht eine Sekundäranalyse von Projekten elektronisch überwachten Hausarrestes in den USA, Großbritannien und Schweden, die sich vor allem mit Aspekten der Zielgruppenplanung auseinanderset/t. Nach einer kritischen Würdigung der Ergebnisse und ihrer Übertragbarkeit auf deutsche Verhältnisse wird, erstmals für die BRD auf der Grundlage empirisch untermauerter Erkenntnisse, die strafrechtsdogmatische und verfassungsrechtliche Problematik einer Implementation dieser Sanktionsalternative in das deutschc Sanktionensystem untersucht.

1999, 278 S., brosch., 40,-DM, 292,- öS, 37,-sFi; [SBN 3-7890-5816-5

(Interdisziplinäre Beiträge zur Kriminologischen Forschung, Bd. 14)

\section{NOMOS Verlagsgesellschaft} 76520 Baden-Baden

22 Vgl. etwa AndrasSajo (1998) Corruption, Clientelism and the Future of the Constitutional State in Eastern Europe, in: Fast European Consitutional Review 7,2, 37-46.

23 Charles deMontesquieu (1734) Considérations sur les causes de la grandeur des Romains et de leur décadence; J.J. Rousseau (1750) Discours sur les sciences et les arts.

24 Fortlaufende Argumentationshilfen auf [http://wow cransparency.de]. 